zwischen den engen Grenzen, welche der Name feststellt, sondern wendet sich auch auf die ïbrigen Theile der granen und weissen Substanz. Von diesen muss die Erkrankung der grauen Substanz als primäre bezeichnet werden, als deren Ausfluss der Faserausfall in den weissen Strängen aufgefasst werden muss. Der Faserausfall der Pyramidenstränge hat seinen Ursprung in der Gehirnrinde, der ibrige Faserausfall bat seine Ursache in der Nervenzellenerkrankung der grauen Substanz, was bereits Marie ausgeführt hat, dessen Ansicht durch meine Ergebnisse bekräftigt wird.

2.

Anmerkungen zu dem Aufsatz: "Ueber die Veränderungen der motorischen Functionen bei Störungen der Sensibilität" von A. v. KonNLLoFF, Privatdocent in Moskau. Deutsche Zeitschrift für Nervenheilkunde. Bd. XII, H. 3 u. 4. 1898.

Von

\title{
Adolf Bickel.
}

Auf dem internationalen medicinischen Congresse zu Moskau hat A. v. Korniloff im August 1897 einen Vortrag mit Demonstrationen über die Veränderungen der motorischen Functionen bei Störungen der Sensibilität gehalten, der jetzt im Heft vom 19. Nai 1898 der dentschen Zeitschrift für Nervenheilknnde im Abdruck vorliegt.

In dieser Arbeit handelt der Verfasser von Versuchen, welche er an Hunden über die Bedeutung der sensiblen Nerven für die Bewegungen der Thiere angestellt hat. Er durchschnitt zu diesem Ende Hunden die sensorischen Nerven für eine Hinterextremität, sei es nur zum Theil, sei es in vollständiger Weise, um eine totale Anästhesie des betreffenden Gliedes zu erreichen, and erhielt die so operirten Thiere monatelang am Leben.

Von einem Hunde, dem die sensiblen Nerven für das linke Bein durchschnitten waren (3., 4., 5., 6., 7. Lumbal- und 1., 2. Sacralwurzel) und der in Folge dessen bis zum Ligamentum Poupartii durchans gefühllos war, giebt v. Kor niloff an, dass am Anfange nach der Operation die betreffende Extremität wie gelähmt war. Zwei und ein halb Monate später aber schreibt er von demselben Versuchsthier:

"Im Grossen und Ganzen muss aber gesagt werden, dass die Anzahl der Bewegungen fast gleich Null ist und dass die wenigen, welche noch geblieben sind, gleichsam atactisch gemacht werden' (1. c. S. 202).

Ferner heisst es auf derselben Seite der Abhandlung:

"Das Kniegelenk befindet sich grösstentheils in Extensionsstellung; wenn der Hund sich erhebt, ist das Bein gerade wie ein Stock".

Aus diesen beiden Sätzen geht unzweidentig hervor, dass das betreffende Versuchsthier $21 / 2$ Monate nach der Operation nur in höchst unvollkommener Weise die Motilität in seinem anästhetischen Bein wiedererlangt hatte. 
Neben diesem Versuche, wo einem Hunde sämm tliche sensiblen Nerven für eine Extremität durchschnitten waren, stellte v. Korniloff noch andere Experimente an, bei denen er nur einen Theil der einem Glied zugehörigen centripetalen Nerven durchtrennte. Er beobachtete an diesen Thieren, dass die motorischen Störungen einer Extremitat um so grösser wurden, je mehr sensible Nerven ausser Function geserzt waren.

Aus allen seinen Experimenten glaubt nun der Verfasser den Schluss ziehen zu dürfen, dass einmal die Motilität eines Gliedes am so mehr gestört wird, je grösser der Sensibilitätsverlust ist, dass andererseits bei Durchschneidung aller zur Extremität gehenden hinteren Wurzeln ,die Extremität beinahe in einen Lähmungszustand" geräth (S. 204).

Diese Experimente v. Korniloff's und ganz besonders die Schlnssfolgerungen, welche der Verfasser aus ihnen zieht, stehen nun einigermassen im Widerspruch mit Resultaten von Versuchen, welche ich an Hunden gewonnen labe (vgl. meine Arbeit: „Ueber den Einfluss der sensiblen Nerven und der Labyrinthe auf die Bewegungen der Thiere". Pflüger's Archiv. Bd. 67. 1897).

Auch bei meinen Versuchsthieren, welchen die sämmtlichen sensiblen Nerven für eine Extremität durchschnitten waren, befand sich das anästhetische Glied anfangs nach der Operation in einer Art von Lähmungszustand; doch konnte ich beobachten, dass sich bei einigen meiner operirten Hunde diese Lähmung in ziemlich hohem Maasse wieder zurückbildete.

Auch ich hatte unter meinen operirten Hunden Exemplare, welche Monate nach der Operation denen ähnelten, die v. Korniloff beschreibt. Aber ich glaube, dass bei allen derartigen Versuchen diejenigen Thiere am beweiskräftigsten sind, welche bei gleichmässig ausgedehnter Anästhesirung am wenigsten von der Norm abweichen.

$Z_{u}$ vollkommen fehlerhaften Vorstellungen über die Function der sensiblen Nerven beim Hunde würde es aber führen, wollte man die Angabe v. Korniloff's, dass die Motilität einer Extremität um so mehr gestört wird, je grösser der Sensibilitätsverlust ist, verallgemeinern und nun etwa schliessen, dass ein Hund, dem z. B. die sensiblen Nerven für beide Hinterbeine durchschnitten wurden, num anch an diesen beiden Extremitäten so $\mathrm{zu}$ sagen gelähmt sein müsste.

Ich habe seiner Zeit darauf hingewiesen, dass Hunde nach unilateraler Durchtrennnng der hinteren Spinalwurzeln für den Hinterkörper verhältnissmässig grössere Störungen dauernder Art zeigen, als solche, welche an beiden Hinterbeinen anästhetisch gemacht wurden. Ich schrieb damals:

„Der Grund dafïr, dass man nach unilateraler Durchtrennung der hinteren Spinalwurzeln verhältnissmässig schlechtere Resultate erzielt and somit viel unvollkommener über die Bedentung der sensiblen Nerven des Körpers aufgeklärt wird, als wenn man doppelseitig die Operation vornimmt, scheint nur zum Theil daran zu liegen, dass die Thiere, welche während der Zeit der Shokwirkung der Operation genöthig sind, auf den drei gesunden Beinen zu gehen, diese Gepflogenheit mehr oder minder beibehalten und darum es der operirten Extremität an der nöthigen Uebung, welche zu ihrem regelrechten Gebrauch nach der Wiederherstellung des Thieres nöthig ist, fehlen lassen. Anders ist es nach dem bilateralen Eingriff. Hier liegt kein Grund vor, der die Thiere bewegen könnte, ein 
Bein zu bevorzugen, da beide die gleiche Schädigung erfahren haben ${ }^{\prime \prime}$ (1. c. S. 314).

Ein Hund, dem man sämmtliche sensiblen Nerven für beide Hinterbeine, wie überhaupt für den ganzen Hinterkörper und den Schwanz durchschnitten hat, ist nun nach meinen Erfahrungen zwar in den ersten Tagen nach der Operation wie gelähmt und lässt bei der Locomotion mit den Vorderbeinen die Hinterextremitäten schlaff nachschleifen, aber schon wenige Wochen nach der Operation hat er - vorausgesetzt, dass die Operation gut ausgeführt war — fast die normale Beweglichkeit in den anästhetischen Gliedmassen wiedererlangt.

Ich habe an der oben genannten Stelle ausführlich geschildert, was ein solches am ganzen Hinterkörper anästhetisches Thier noch zu leisten im Stande ist, wie es vortrefflich laufen und Treppen steigen kann, wie es mit Sicherheit Hindernisse überspringt, ja wie es anf den Sohlen der gefïhllosen Hinterbeine tanzt und sich in die Luft schnellt bei aufgerichtetem Körper, um ein vorgehaltenes Stück Speck zu erhaschen.

Ich besass einen derartig operirten Hund, welcher besonders zahm and zutraulich war. Trat ich des Morgens an seinen Käfig, um ihm Futter zn reichen, so lief er am Gitter hin und her und wedelte lebhaft mit seinem total gefühllosen Schwanze, genau so, wie es ein normales Thier gethan haben würde.

Das Alles beweist denn doch, dass die Function der sensiblen Nerven nicht so indispensabel für die Motiliät der von uns untersuchten Körpertheile beim Hunde ist, wie man nach der Lectïre der Arbeit v. Korniloff's annehmen könnte.

Das ist ja richtig, dass die Motilität überhaupt an die Sensibilität gebunden ist, dass, wenn man einem Thiere alle centripetalen Nerven durchschnitte, das Thier vollkommen gelähnt wäre; aber ich kann auf Grund meiner Beobachtungen nicht zugeben, dass nach Durchschneidung der sensiblen Nerven für einzelne Extremitäten diese Körpertheile einem danernden Lähmungszustand beim Hunde anheimfallen, wie es v. Kornil off zu behaupten scheint. - Anch bei diesen Untersuchungen sind die Thiere am beweiskräftigsten, welche nach gleichem Eingriff die geringsten Störungen zeigen. - Die klinische Erfahrung spricht gleichfalls gegen v. Korniloff's Anschauung.

Wie liesse sich die Beobachtung erkiären, dass Tabeskranke nach völligem Schwund der Sensibilität, z. B. an den Beinen, häufig kaum die geringfügigsten Abnormitäten beim Gebrauch dieser Glieder anfweisen? Nach v. Korniloff's Erfahrungen am Hunde müssten diese Patienten gelähmt sein.

Nicht einen Ausfall, ein Minus an Bewegungen, kann man an den Hunden mit gefühllosem Hinterkörper so sehr beobachten, im Gegentheil ein gewisses Uebermaass motorischer Leistungen macht sich viel eher bei ihnen geltend. So habe ich häufig Gelegenheit gehabt, constatiren zu können, dass, während ein solches Thier ruhig dasass, die gefübllosen Hinterbeine oder auch der Schwanz unaufhaltsam kleine Bewegungen ausführten (unfreiwillige Spontanbewegungen?), die nicht anders zu deuten waren, als bedingt durch eine nach Durchschneidung der sensiblen Nerven ausfallende Hemmung beim Gebrauch der motorischen Apparate dieser Körperregion. 